\title{
Correction to: A multi-target approach for discovery of antiviral compounds against dengue virus from green tea
}

\author{
Pooja Mahajan ${ }^{1} \cdot$ Shantanu Tomar ${ }^{2} \cdot$ Adarsh Kumar $^{1} \cdot$ Neelesh Yadav $^{3} \cdot$ Aditya Arya $^{1} \cdot$ Vivek Dhar Dwivedi $^{1}$
}

Published online: 23 July 2020

(c) Springer-Verlag GmbH Austria, part of Springer Nature 2020

\section{Correction to: \\ Network Modeling Analysis in Health Informatics and Bioinformatics (2020) 9:20 \\ https://doi.org/10.1007/s13721-020-0222-4}

In results and discussion section, the description of figures was revised as follows. In the case of NS1-Compound-1 complex, consider an additional contact of Glu12 (Fig. 1a), in case of NS1-Compound-2 complex, six instead of four residues were involved in contact (Fig. 1b). In case of RDRP-Compound-1 complex, seven instead of three hydrogen bonds were formed (Fig. 1d), while in case of RDRP Compound-2 complex nine instead of seven hydrogen bonds were recorded (Fig. 1e). In case of MTD-complex 1 consider an addition contact of Leu17 (Fig. 1g).

The original article can be found online at https://doi.org/10.1007/ s13721-020-0222-4.

Aditya Arya

contact.adityarya@gmail.com

$\triangle$ Vivek Dhar Dwivedi

vivek_bioinformatics@yahoo.com

1 Center for Bioinformatics, Computational and Systems Biology, Pathfinder Research and Training Foundation, Knowledge Park-III, Greater Noida, India

2 Department of Biotechnology, GLA University, Mathura, India

3 Information Technology Cell, Tropical Forest Research Institute, Jabalpur, India
Publisher's Note Springer Nature remains neutral with regard to jurisdictional claims in published maps and institutional affiliations. 